\title{
Query By Attention: Visually Searchable Information Maps
}

\author{
Mark A. Foltz \& Randall Davis \\ Artificial Intelligence Laboratory \\ Massachusetts Institute of Technology \\ Cambridge, Massachusetts 02139 \\ http://www.ai.mit.edu
}

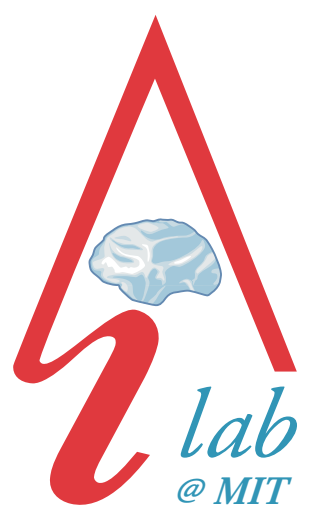

The Problem and Motivation: Vast amounts of useful information are accessible to us in textual databases, yet we prefer graphs, charts, and maps to interpret this information and make decisions. One advantage of these graphical presentations is that the user can query by attention - answer questions by controlling visual attention (and receiving immediate feedback), rather than manipulating a database query interface and waiting for a server to return results. The user can more rapidly explore the information space because he needs to alter only his visual attention (and not the interface) to adjust the parameters of the query.

The particular kind of presentation we consider are information maps, which spatially array graphical symbols representing items of information and their attributes. We propose a method for designing visually searchable maps based on experimental results about what kinds of visual search are easy. Ideally, their users should be able to do query by attention, answering questions about the information quickly by controlling visual attention (i.e., through spatial selection and visual search), instead of manipulating an interface. More detail about this work can be found in [2].

Previous Work: Pirolli et al. have performed a study that tracks the eye movements of users as they perform textual navigation in a hyperbolic tree [3]. Their theory focuses on how semantic (textual) cues serially guide the attentional spotlight, while our work focuses on perceptual, preattentive visual properties that the viewer can process in parallel. Clearly these processes are complementary and research to integrate such models is needed.

Other interfaces provide interactive methods for rapidly filtering data in visualizations [1]. These methods can be combined with query by attention to manage large data sets, letting the user do both interactive filtering and attention-directed visual search.

Approach: Our approach focuses on the visual properties that the human visual system can filter rapidly and preattentively. Vision science tells us that these properties include shape, color, motion, and depth, among others [4]. We can, in addition, direct our attentional spotlight to focus on a contiguous spatial region of the display.

The task then is to map the information to be visualized onto these capabilities for attentional filtering. We have developed and implemented a design flowchart that, given a base of information and set of queries users are likely to make, maps the attributes of the information to visual properties of the display. This flowchart embodies a design process for creating visually searchable information maps, and summarizes the ways we can efficiently perform visual search, including conjunction searches. Figure 1 shows a use of the design flowchart and an information map created from the resulting design.

Effective information maps also require consideration to be given to the properties of the visual stimuli in the map, to ensure that they are far enough apart in perceptual space to permit effective attentional filtering. For example, work in human vision indicates that one out of up to nine colors may be searched for efficiently, provided the colors are far apart and linearly separable in perceptual color space.

Impact: Visual search for information is preferred because it is better suited for people, while database search remains better suited for computers. Information maps, which give meaning to a item's location, also engage their viewers' capabilities for spatial imagery and spatial memory. If the user remembers an item's location, he can return to the map and find it there, instead of repeating a textual query or scanning a list.

Efficient visual search in information maps is not possible in all cases. But maps that better employ our visual perception potentially have a great advantage over textual interfaces for understanding and managing information. We believe that visual information tools can benefit from progress in vision science, by offering design guidance 

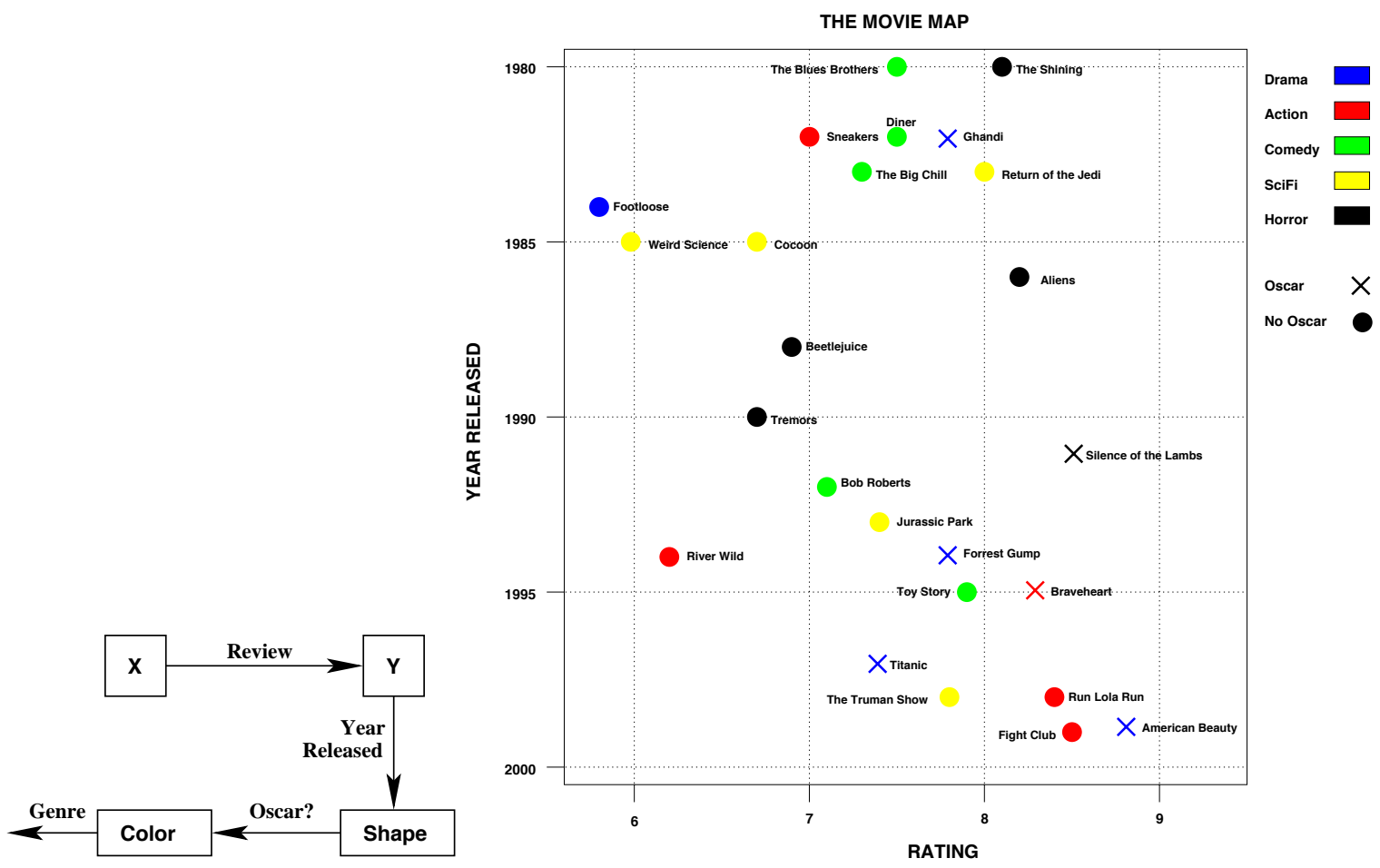

Figure 1: Left, a design for the movie map (shown as a path in the flowchart found in [2]). Right, the resulting information map. (Try finding the horror movie that won an Oscar.)

related to our perceptual capabilities.

Future Work: We are developing a software tool that, given the data to be plotted, can produce a visualization like that in Figure 1. An early prototype of the tool is able to guess the information types of fields in the data, map them to visual properties, and produce an initial information map.

We plan to add interactive features (details-on-demand, filtering, text search) to these maps. In the future we will address scalability, both in terms of the number of information items to visualize at once, and the number of attributes of each item. The long-term goal is to ask the user how she wants to interpret the information in her own terms - i.e., "Show me how Oscars relate to genres" - and revise the information design accordingly, so that those relationships become perceptually evident.

Research Support: This research is sponsored by the MIT Ford Motor Company Collaboration and MIT Project Oxygen.

\section{References:}

[1] Christopher Ahlberg and Ben Shneiderman. Visual information seeking: Tight coupling of dynamic query filters with starfield displays. In Proceedings of CHI '94: Human Factors in Computing Systems, pages 313-317, Boston, Massachusetts, 1994.

[2] Mark A. Foltz and Randall Davis. Query by attention: Visually searchable information maps. In Proc. Fifth International Conference on Information Visualisation, London, 2001.

[3] Peter Pirolli, Stuart K. Card, and Mija M. Van Der Wege. Visual information foraging in a Focus+Context visualization. In Proceedings of CHI 2001, Seattle, Washington, 2001.

[4] Jeremy M. Wolfe. Visual search. In H. Pashler, editor, Attention. University College London Press, London, 1996. 